\title{
GLACIER FRONTAL LINE EXTRACTION FROM SENTINEL-1 SAR IMAGERY IN PRYDZ AREA
}

\author{
Fei Li ${ }^{1, *}$, Zhenling Wang ${ }^{1}$, Shengkai Zhang, ${ }^{1}$ Yu Zhang ${ }^{1, *}$ \\ ${ }^{1}$ Chinese Antarctic Center of Surveying and Mapping, Wuhan University, 430079, China \\ -fli@whu.edu.cn, yuzhang_spl@whu.edu.cn
}

Commission III, WG III/9

KEY WORDS: SAR, Glacier Frontal, Sentinel-1, CFAR, Profile analysis

\begin{abstract}
:
Synthetic Aperture Radar (SAR) can provide all-day and all-night observation of the earth in all-weather conditions with high resolution, and it is widely used in polar research including sea ice, sea shelf, as well as the glaciers. For glaciers monitoring, the frontal position of a calving glacier at different moments of time is of great importance, which indicates the estimation of the calving rate and flux of the glaciers. In this abstract, an automatic algorithm for glacier frontal extraction using time series Sentinel-1 SAR imagery is proposed. The technique transforms the amplitude imagery of Sentinel-1 SAR into a binary map using SO-CFAR method, and then frontal points are extracted using profile method which reduces the $2 \mathrm{D}$ binary map to $1 \mathrm{D}$ binary profiles, the final frontal position of a calving glacier is the optimal profile selected from the different average segmented profiles. The experiment proves that the detection algorithm for SAR data can automatically extract the frontal position of glacier with high efficiency.
\end{abstract}

\section{INTRODUCTION}

Glacier is the sensitive indicators of global environmental change. In recent years, glaciers have melted faster than expected, leading to greater impacts on sea level rise. Exploring the relationship between glaciers melting and climate change is important in explaining the past, monitoring present, predicting the future sea level rise and climate change (Warren and Aniya, 1999). The recent research indicates that the mass loss from glacier and ice sheets contributes nearly one third of the current sea level rise (Strozzi et al., 2017). Especially in polar regions, the frontal ablation of tidewater is the largest part of glacier mass loss (Khan et al., 2015; McNabb et al., 2015), for example, calving of icebergs and frontal melt. The increase archived SAR satellite opened up new possibilities for monitoring and detect the relationship between the glacier mass loss and sea level rise. Calving is the process in which ice blocks separate from a glacier's margin (Cuffey and Paterson, 2010). Iceberg calving is an important cause of the mass loss of glaciers and can be expressed as calving rate and calving flux (Benn et al., 2007). To understand the mass loss of glaciers, the accurate glacier frontal position is very important. At the beginning, glacier frontal is treated as the boundary between relative homogenous areas, and it can be extracted using edge detectors such as canny and sobel (Seale et al., 2011). Optical satellite including MODIS and Landsat can provide the plentiful contexture information, which serves as the feature descriptor for glacier frontal between ice and water. However, during winter season, the optical image is bad in quality due to Night phenomenon (Kachouie et al., 2012). In the last 30 years, the Synthetic Aperture Radar (SAR) technique has been widely used for remote sensing of the Earth surface (Moreira et al., 2013). SAR imagery can provide high-resolution data independent from daylight, cloud coverage and weather conditions. Local adaptive thresholding method developed from Radarsat-1 imagery has an average accuracy of larger than $100 \mathrm{~m}$, which may lead to a huge uncertain for mass loss calculation (Liu and Jezek, 2004b). the earliest profile method for the automatic derivation of glacier frontal position from all available Landsat data was developed by Rosenau(2013). Later, the improved and extended profile method was applied to the derivation of glacier frontal position from the high resolution SAR images (Han et al., 2016). Moreover, the newly launched Sentinel-1 satellite can provide higher resolution of SAR imagery, so a higher spatial resolution of glacier frontal is expected.

In this abstract, the glacier frontal extraction method using Sentinel-1 SAR imagery is investigated. We first transform the amplitude SAR imagery into binary map using SO-CFAR approach then the profile analysis method is carried out to obtain the frontal point of each profile line. Moreover, the choice of profile number is also discussed, The accuracy of the frontal position detection through this detection method is evaluated by comparing the detected frontal points to those obtained from manually tracking the frontal line in the ArcGIS software, then comparing to select the optimal profile number is discussed. Finally, in the same area, comparing with the previous profile method to prove the superiority of the method.

\section{METHODOLOGY}

In this abstract, the method of automatically determine outlet glaciers frontal positions using Sentinel-1 SAR data is based on

* Corresponding authors 
the analysis of the binary value distribution along defined profiles which cover the transition zone between the water and the glacier after transforming the amplitude imagery of Sentinel1 SAR into a binary map. The detection system includes two main steps: Binary map using CFAR method, Glacier frontal detection. Finally, the ice/water transition line is obtained as a vector file by automatic connection of the detected profile points. The details of these two main steps are explained in the corresponding subchapters.

\subsection{Binary map using CFAR method}

According to the backscattering mechanism of SAR, we first transform the Sentinel-1 data into a binary map using SO-CFAR method. Considered of the traditional mean value and statistic CFAR detection algorithm, firstly, the background clutter pixels of every local sliding window are modified using local filtering, so that the speckle of the SAR image is decreased. Then, the distribution parameters of the clutter are estimated from the filtered image. At last, a SO-CFAR detector is used to obtain the binary result. In this study, we set 1 for the glacier pixel and -1 for open water area.

\subsection{Glacier frontal detection}

The second step of the profile method is the data processing of the binary map, which can be further divided into three substeps: limiting the search area, generating profiles in the search area, extracting binary values along profiles. The details are given in the following contents.

During this period both the frontal position and glacier margins changed. In order to process all data more quickly and simplify the extraction of the glacier frontal, the side borders of the glacier were drawn and kept constant for the entire time series when in Time Series Analysis. Therefore, the limiting side borders should be always on the glacier for the entire time series considered but they must not match exactly the real side borders of the glacier on a certain date. we first limit the approximate edge line of the glacier manually digitized in the ArcGIS software and then analyse the profile along the direction of the edge line, so as to convert the two-dimensional transformation of the glacier into a one-dimensional problem.

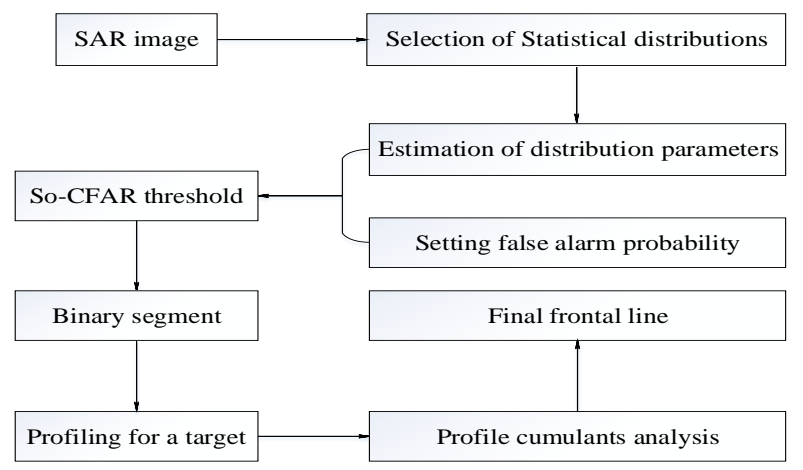

Figure 1. Workflow of extraction of the glacier frontal line

After the glacier edge line were determined, a series of interpolated curves can be generated within these edge lines. For improving the accuracy of front line detection, we divide the edge line region into several small sections by the method of equidistant separation. Then the front point is obtained by calculating its position on each section line. In our work, the front point is determined by calculating the accumulative value of the binary map. We mean that the corresponding points with maximum cumulative value is evaluated as the front point for each section line, and the final frontal line is obtained using linear fitting method. Specific algorithm flow as shown in Figure 1.

\section{EXPERIMENT AND ANALYSIS}

This part mainly tells the study area and experimental data. Secondly, the final frontal position of a calving glacier is the optimal profile selected from the different average segmented profiles. Finally, in the same area, comparing with the previous profile method to prove the superiority of the method in this paper.

\subsection{Study area and experimental data}

The glacier frontal line extraction experiment is conducted on Sentinel-1 IW SLC HH imagery in Prydz Bay area $\left(69^{\circ} 2^{\prime} \mathrm{S}, 77^{\circ} 51^{\prime} \mathrm{E}\right)$, it is acquired on July 26,2016 , with the resampled resolution of $15 \mathrm{~m}$, and its image size is $3956 * 5021$ pixels, and the validation data is obtained using manual labeling, seen in Figure 2.

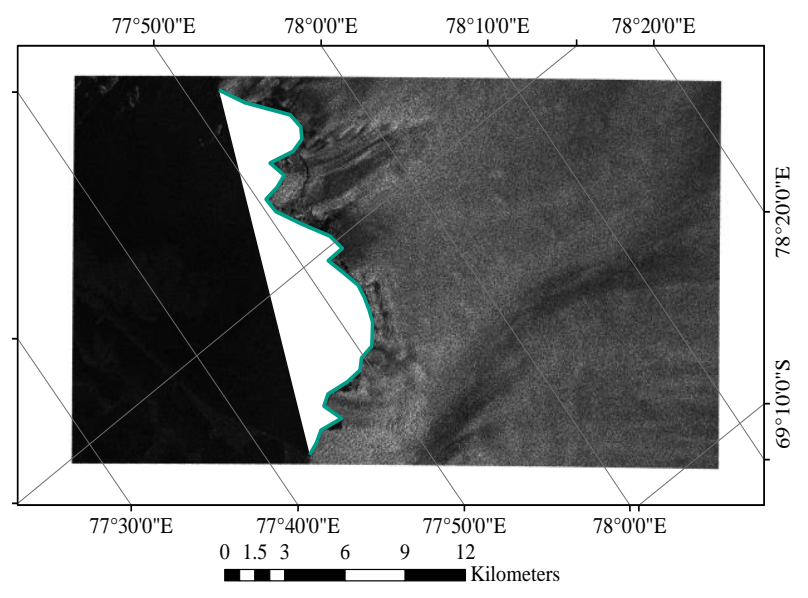

Figure 2. Study Area and experimental data

\section{2 Selection of optimal segmentation}

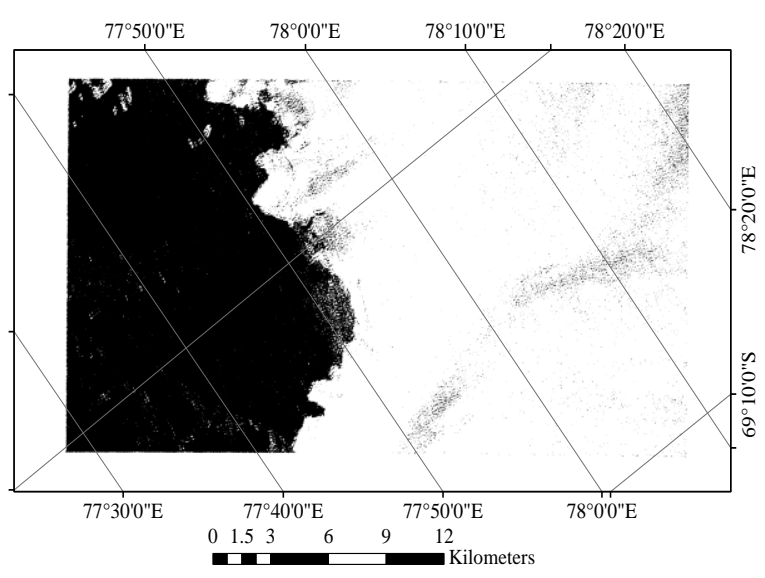

(a) Binary map using So-CFAR 
The International Archives of the Photogrammetry, Remote Sensing and Spatial Information Sciences, Volume XLII-3, 2018 ISPRS TC III Mid-term Symposium “Developments, Technologies and Applications in Remote Sensing”, 7-10 May, Beijing, China

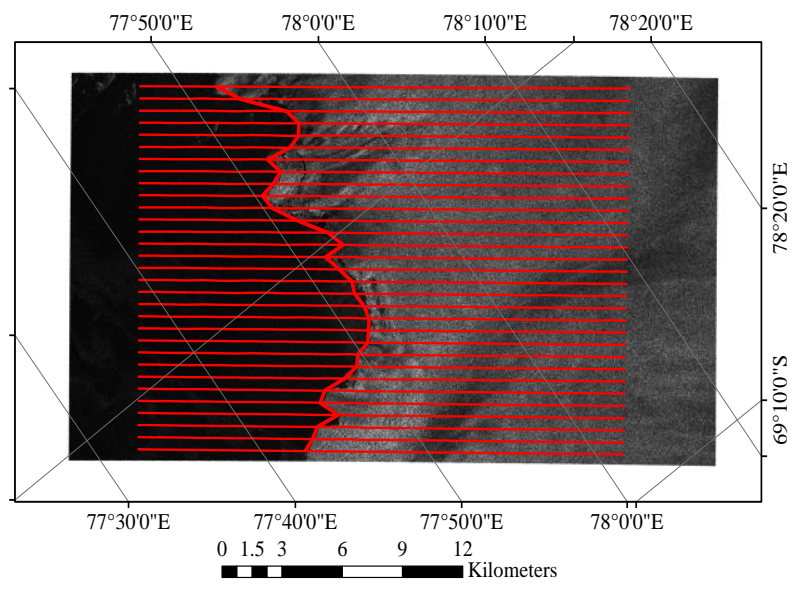

(b) Results

Figure 3. Glacier frontal line extraction results

Before selecting the optimal segmentation, The technique transforms the amplitude imagery of Sentinel-1 SAR into a binary map using CFAR method, and then frontal points are extracted using profile method which reduces the 2D binary map to 1D binary profiles for automatic detection frontal points efficiently, The results of binary map using CFAR method and glacier frontal line using profile analysing are shown in Figure 3.

Moreover, we find that the choice of section numbers effects the accuracy of frontal line detection in the experiment. For selection the best parameter, we set the section number from 10 to 60 .

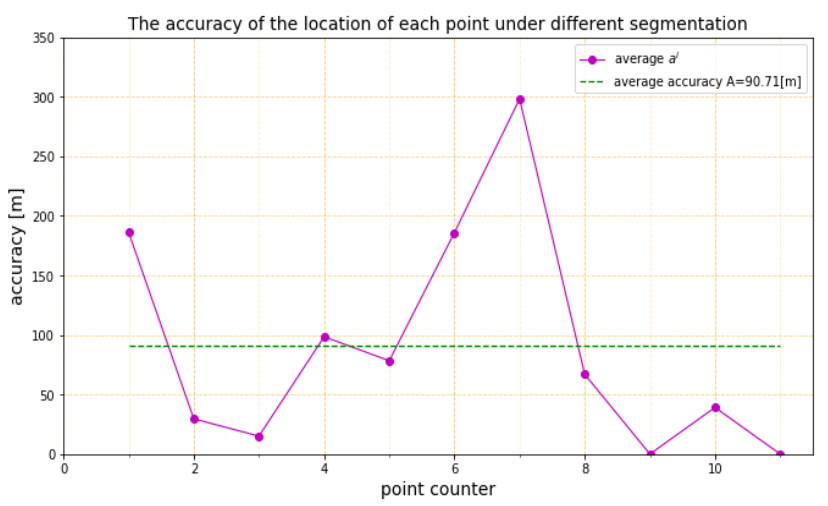

(a) 10 Profiles

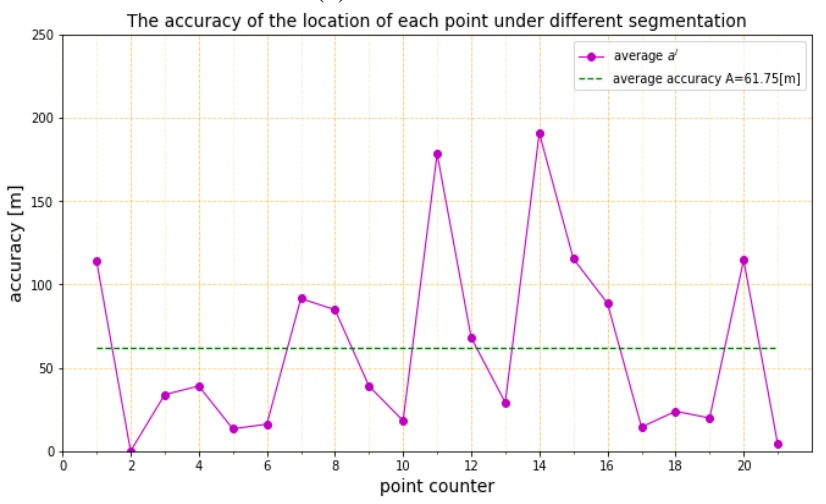

(b) 20 Profiles

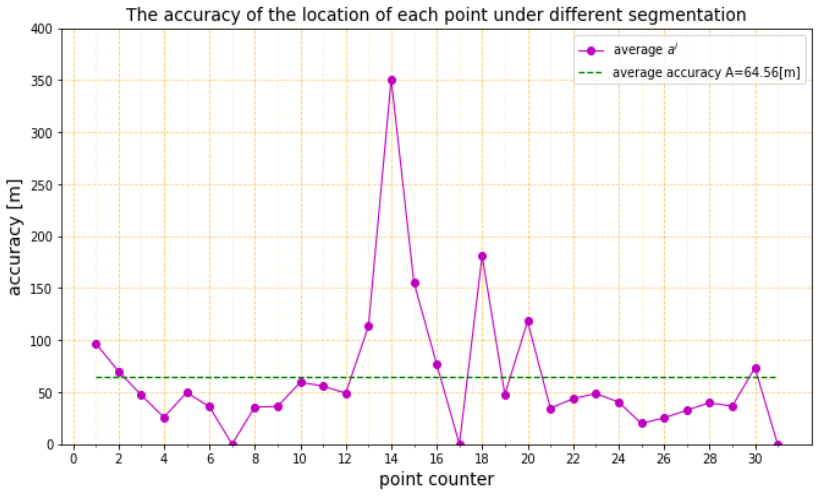

(c) 30 Profiles

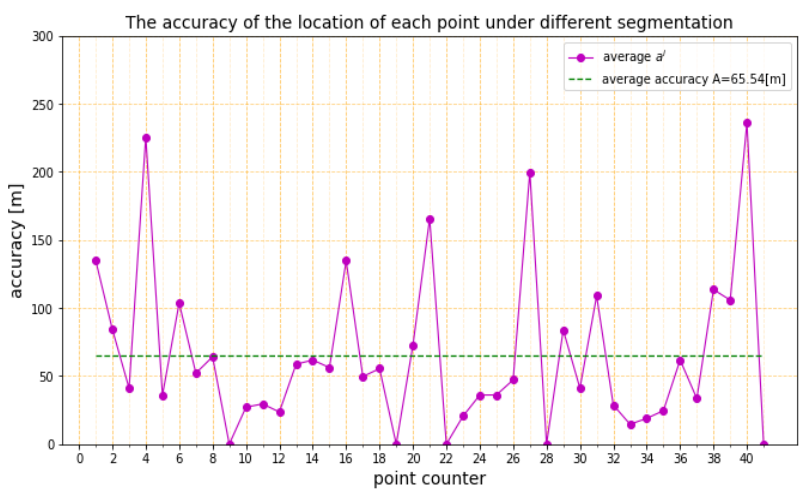

(d) 40 Profiles

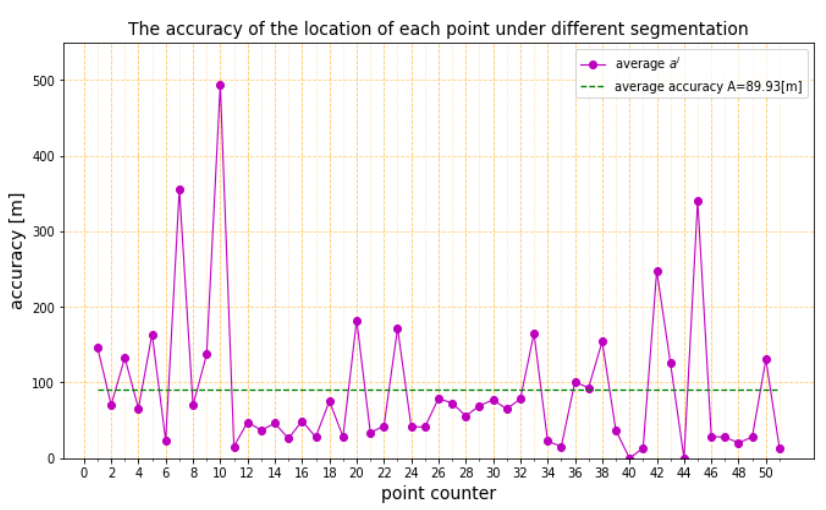

(e) 50 Profiles

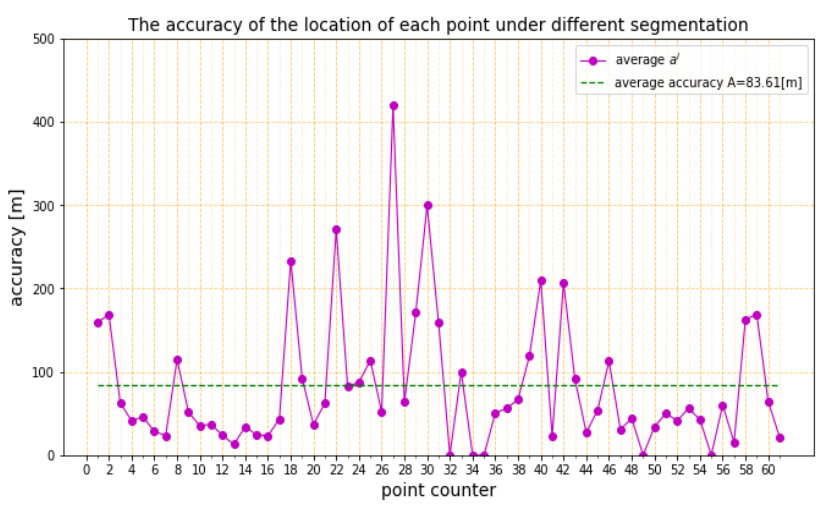

(f) 60 Profiles 


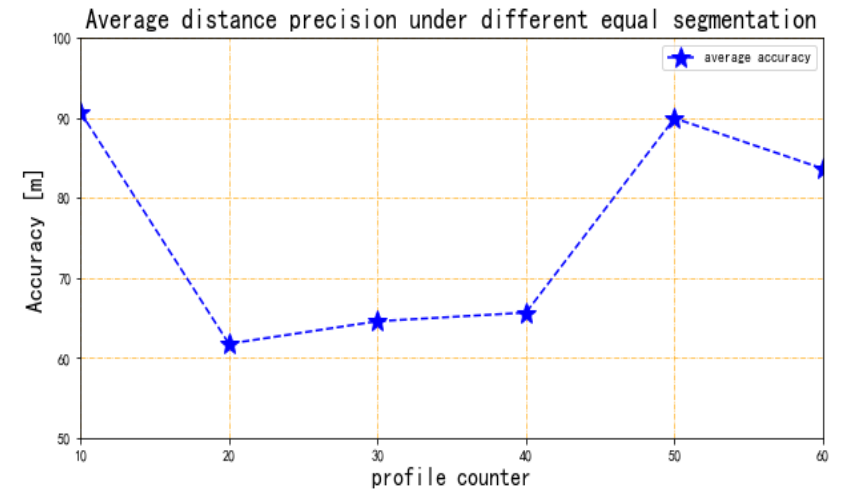

(g) Comparison of different numbers of Profile

Figure 4. Average accuracy of different numbers of profile

The accuracy of the frontal position detection through this detection method is estimated by comparing the detected frontal points to those obtained from manually tracing the frontal line in the ArcGIS software, and compared the average accuracy under different selection. The average accuracy means the distance between detected frontal points and the corresponding labelled frontal points. the results are shown in Table 1 and Figure 4.

\begin{tabular}{ccc}
\hline Number of Profile & $\begin{array}{c}\text { Distance between } \\
\text { each profile/m }\end{array}$ & Average Accuracy/m \\
\hline 10 & 1600 & 90.71 \\
20 & 800 & 61.75 \\
30 & 533 & 64.56 \\
40 & 400 & 65.64 \\
50 & 320 & 89.93 \\
60 & 267 & 83.61 \\
\hline
\end{tabular}

Table 1. Accuracy of glacier frontal detection using different number of profile

The result in Figure 4 and Table 1 indicates that 20 profiles (distance between each section is $800 \mathrm{~m}$ ) can obtain the best results with the average accuracy of $61.75 \mathrm{~m}, 30$ profiles (distance between each section is $533 \mathrm{~m}$ ) can obtain the Second better precision with the average accuracy of $64.75 \mathrm{~m}$, Comparing with the 20 profiles, the 30 profiles can ensure the integrity and details of the front contour of the glacier. while 10 profiles obtain the lowest accuracy of $90.71 \mathrm{~m}$. Since the resolution of the Sentinel-1 imagery is $15 \mathrm{~m}$, which means that the proposed method can obtain the glacier frontal line with the average error of less than six pixels.

The experimental results indicate that the user can choose the optimal segmentation based on the information obtained from the different contour extracted from glacier frontal position.

\section{3 Correlation contrast experiment}

In order to illustrate and verify that the method is superior to other methods, The contrast experiment is in the same area and uses the same data, adopting previous profile method which was provided with highest precision, when applied in Sentinel-1
SAR data (Han et al., 2016). The accuracy of the frontal position detection through this contrast experiment detection method is estimated by comparing the detected frontal points to those obtained from manually tracing the frontal line in the ArcGIS software, similarly. Finally, calculating the average accuracy of the comparison experiment under different profiles. The contrast experiment results are shown in Figure 5 and Figure 6.

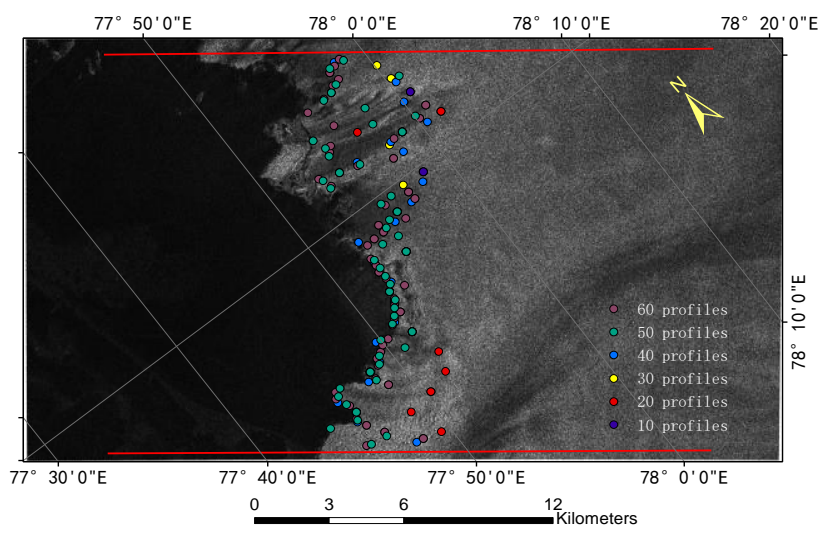

(a) The distribution of glacier frontal points using previous profile method

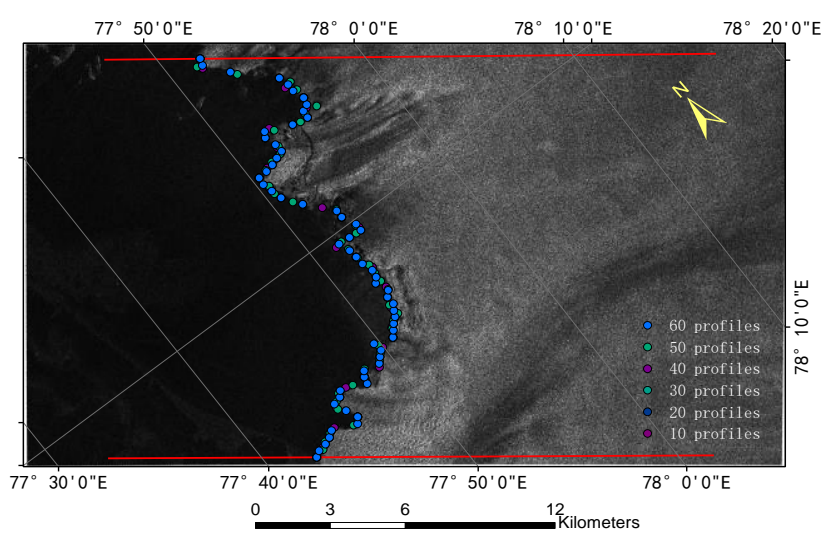

(b) The distribution of glacier frontal points using the method of this paper

Figure 5. Experimental results of different methods

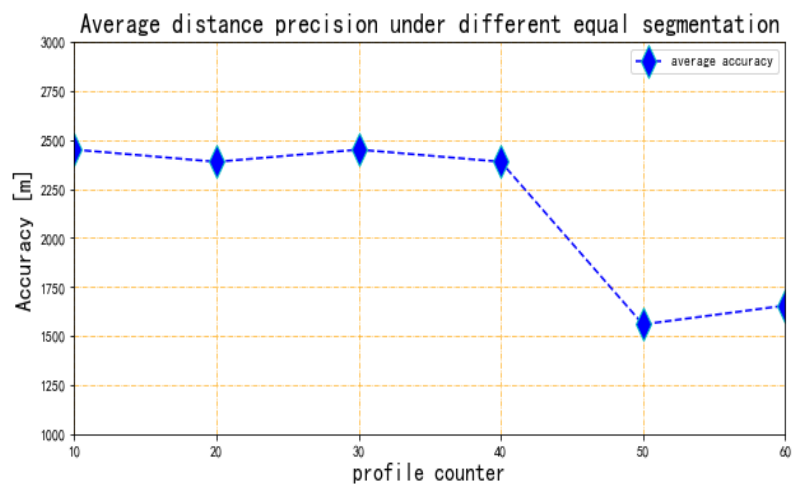

Figure 6. Comparison of different numbers of Profile 
From Figure 5, it can be seen by artificial vision is that the glacier frontal points detected using previous profile method is much more offset by the position of the true glacier contour. However, the locations of glacier frontal points detected using the method introduced in this paper basically coincide with the true glacier frontal position, with the accuracy of the information is shown in Table 1 and Figure 4. Figure 6 describes the average accuracy of the contrast experiment at different profiles, it can be seen that the average accuracies of frontal position by contrast experiment using previous profile method are very larger than using the proposed method of this paper. As a whole, all the average accuracies are above $1500 \mathrm{~m}$, and the maximum is $2500 \mathrm{~m}$, Since the resolution of the Sentinel-1 imagery is $15 \mathrm{~m}$, which means that the proposed method can obtain the glacier frontal line with the average error of more than 100 pixels. According to the image map of the experimental area, the previous profile method has great limitation in the case of water on the glacier surface. Therefore, the proposed method of this paper has certain superiority and wide applicability.

\section{CONCLUSION}

In this abstract, an automatic glacier frontal extraction method is proposed. We first transform the amplitude SAR imagery into binary map using SO-CFAR algorithm, then the profile analysing approach is used to calculate the frontal point of each section, finally linear fitting procedure is carried out to obtain the final results. In the experiment part, the proposed algorithm is tested on Sentinel-1 SAR imagery in Prydz area. The results indicate that we can obtain the best results when the distance of nearby profile is $800 \mathrm{~m}$, and the average of our method is less than 6 pixels. The contrast experiment proves that the proposed method for SAR data can automatically extract the frontal position of glacier with high superiority and wide applicability. With the free supply of sentinel-1 data ,this method can be applied to large time series and replace the manually mapping.

\section{ACKONWLEDGEMENT}

This study is supported by the National Key R\&D Program of China, No. 2017YFA0603104, the State Key Program of National Natural Science of China, No. 41531069, the fundamental Research Funds for the Central University, No. 2042018kf0050, the China Postdoctoral Science Foundation, No. 2017M612512.

\section{REFERENCES}

Benn, D. I., Warren, C. R., and Mottram, R. H., 2007. Calving processes and the dynamics of calving glaciers. Earth-Science Reviews 82(3), pp. 143-179.

Cuffey, K. M. and Paterson, W. S. B., 2010. The physics of glaciers: Academic Press.

Han, L., Floricioiu, D., Baessler, M. and Eineder, M., 2016. An algorithm for the detection of calving glaciers frontal position from TerraSAR-X imagery. Geoscience and Remote Sensing Symposium IEEE, pp. 6171-6174.
Khan, S. A., Aschwanden, A., Wahr, J. and Kjeldsen, K. K., 2015. Greenland ice sheet mass balance: a review. Reports on Progress in Physics 78(4), pp. 046801.

Kachouie, N. N., Huybers, P. and Schwartzman, A., 2012. Localization of mountain glacier termini in Landsat multispectral images. Pattern Recognition Letters 34(1), pp. 94-106.

Liu, H. and Jezek, K. C., 2004b. A complete high-resolution coastline of Antarctica extracted from orthorectified Radarsat SAR imagery. Photogrammetric Engineering \& Remote Sensing 70(5), pp. 605-616.

Mcnabb, R. W., Hock, R. and Huss, M., 2015. Variations in Alaska tidewater glacier frontal ablation, 1985-2013. Journal of Geophysical Research Earth Surface 120(1), pp. 120-136.

Moreira, A., 2013. Digital beamforming: A paradigm shift for spaceborne SAR. Paper presented at the Radar Symposium (IRS), 2013 14th International, pp.23-36.

Rosenau, R., 2013. Untersuchung von Fließgeschwindigkeit und Frontlage der großen Ausflussgletscher Grönlands mittels multitemporaler Landsat-Aufnahmen. (PHD thesis), Technical University of Dresden.

Strozzi, T., Kääb, A. and Schellenberger, T., 2017. Frontal destabilization of Stonebreen, Edgeøya, Svalbard. Cryosphere 11(1), pp. 1-29

Seale, A., Christoffersen, P., Mugford, R. I. and O'Leary, M., 2011. Ocean forcing of the Greenland Ice Sheet: Calving fronts and patterns of retreat identified by automatic satellite monitoring of eastern outlet glaciers. Journal of Geophysical Research Earth Surface, 116(F3).

Warren, C. and Aniya, M., 1999. The calving glaciers of southern South America. Global and Planetary Change 22(1), pp. 59-77. 